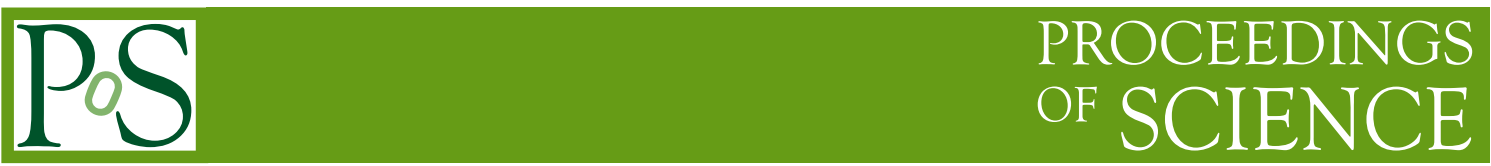

\title{
Color screening in $2+1$ flavor QCD at large distances
}

\author{
Sebastian Steinbeißer* \\ Physik Department \\ Technical University of Munich \\ James-Franck-Str. 1 \\ 85748 Garching, Germany \\ E-mail: sebastian. steinbeisser@tum.de \\ Johannes Heinrich Weber \\ Department of Computational Mathematics, Science and Engineering (CMSE) \\ Michigan State University \\ E-mail: weberjo8@msu.edu
}

\section{(for TUMQCD Collaboration)}

\begin{abstract}
We study correlation functions of spatially separated static quark-antiquark pairs in $2+1$ flavor QCD in order to investigate the nature of color screening at high temperatures. We perform lattice calculations in a wide temperature range, $116 \mathrm{MeV} \leq T \leq 5814 \mathrm{MeV}$, using the highly improved staggered quark (HISQ) action and several lattice spacings to control discretization effects. We alleviate the UV noise problem through the use of four dimensional hypercubic (HYP) smearing, which enables the reconstruction of correlators and determination of screening properties even at low temperatures and at large distances.

This proceeding contribution is a report on work in progress aimed at improving previous results [1] and prepares a forthcoming publication [2].
\end{abstract}

XIII Quark Confinement and the Hadron Spectrum - Confinement2018

31 July - 6 August 2018

Maynooth University, Ireland

${ }^{*}$ Speaker. 


\section{Introduction}

At zero temperature a heavy quark and antiquark pair forms a bound state (quarkonium state) or a pair of heavy-light mesons. At sufficiently high temperatures $\left(T>T_{c}\right.$ ), (heavy) quarks are not confined anymore due to color screening and collisions with the medium. The Polyakov loop and the Polyakov loop correlator are the order parameters of this confinement-deconfinement transition from hadrons to a quark gluon plasma (QGP) in pure Yang-Mills theory and still provide sensitive probes in full QCD. The continuum limit of the corresponding quark-antiquark free energies have been studied previously (see Ref. [1]) and it has been found that their short distance behavior can be understood in terms of weak-coupling effective field theory calculations in the framework of pNRQCD and EQCD (see Ref. [3]). These studies have been limited by the exponential drop of the signal to noise ratio, which we can now alleviate through the use of smeared gauge fields. We demonstrate that through subtraction of the squared expectation value of the appropriately smeared Polyakov loop we can reconstruct the subtracted free energies at sufficiently large distances and smoothly connect results with different amounts of HYP-smearing (see Ref. [4]) applied. We use four dimensional HYP-smearing throughout this work. This allows a quantitatively predictive study even in the asymptotic screening regime.

We perform lattice calculations in a wide temperature range, $116 \mathrm{MeV} \leq T \leq 5814 \mathrm{MeV}$, using the highly improved staggered quark (HISQ) action (see Ref. [5]) and the MILC code (see Ref. [6]) and several lattice spacings in $2+1$ flavor QCD.

The $Q \bar{Q}$ color average and color singlet (subtracted) free energies are given in terms of the respective (subtracted) Polyakov loop correlators by

$$
F_{Q \bar{Q}}^{(\text {sub. }}=-T \ln \left(C_{P}^{(\text {sub. })}\right), \quad F_{\mathrm{s}}^{(\text {sub. })}=-T \ln \left(C_{\mathrm{s}}^{(\text {sub. })}\right) .
$$

The bare (unsubtracted) Polyakov loop correlators are given by

$$
C_{P}(r)=\left\langle P(0) P^{\dagger}(r)\right\rangle, \quad C_{\mathrm{s}}(r)=\frac{1}{3}\left\langle\operatorname{tr} W(0) W^{\dagger}(r)\right\rangle .
$$

The Polyakov loop $P$ being the trace of a temporal Wilson line $W$, where the latter is not gauge invariant; we define $C_{\mathrm{s}}$ in Coulomb gauge.

$$
P(r)=\frac{1}{3} \operatorname{tr} W(r), \quad W(r)=\prod_{\tau / a=1}^{N_{\tau}} U_{0}(\tau, r),
$$

with $W$ describing the propagation of a static quark via the link variables $U_{0}$. The expectation value of the Polyakov loop averaged over the spatial volume of the lattice, $L=\langle P(r)\rangle$, is used to normalize Polyakov loop correlators

$$
C_{P}^{\text {sub. }}(r)=\frac{C_{P}(r)}{L^{2}}, \quad C_{\mathrm{s}}^{\text {sub. }}(r)=\frac{C_{\mathrm{s}}(r)}{L^{2}} .
$$

This normalizes the correlator $C^{\text {sub. }}$ such that the corresponding free energy $F^{\text {sub. }}$ vanishes at infinite separation in infinite volume. It is important to note that the correlator and $L$ need to be computed for the same regularization, i.e., with the same HYP-smearing level. The subtracted free energies defined in terms of the subtracted correlators are divergence free and have a well-defined continuum 
limit. At distances $r \gtrsim 1 /(g T)$, where $g T \sim m_{\mathrm{D}}$ is the Debye mass, the dimensionally reduced EFT called electrostatic QCD (EQCD) is suitable to describe these correlators (see Ref. [7]). For distances $r \ll 1 /(g T)$, the correlators are sensitive to the inherent non-perturbative magnetic sector and receive contribution from the magnetic mass $\propto g^{2} T$. Hence, a non-perturbative approach is required for studying the large distance behavior.

\section{Screening functions for color singlet and color average free energies}
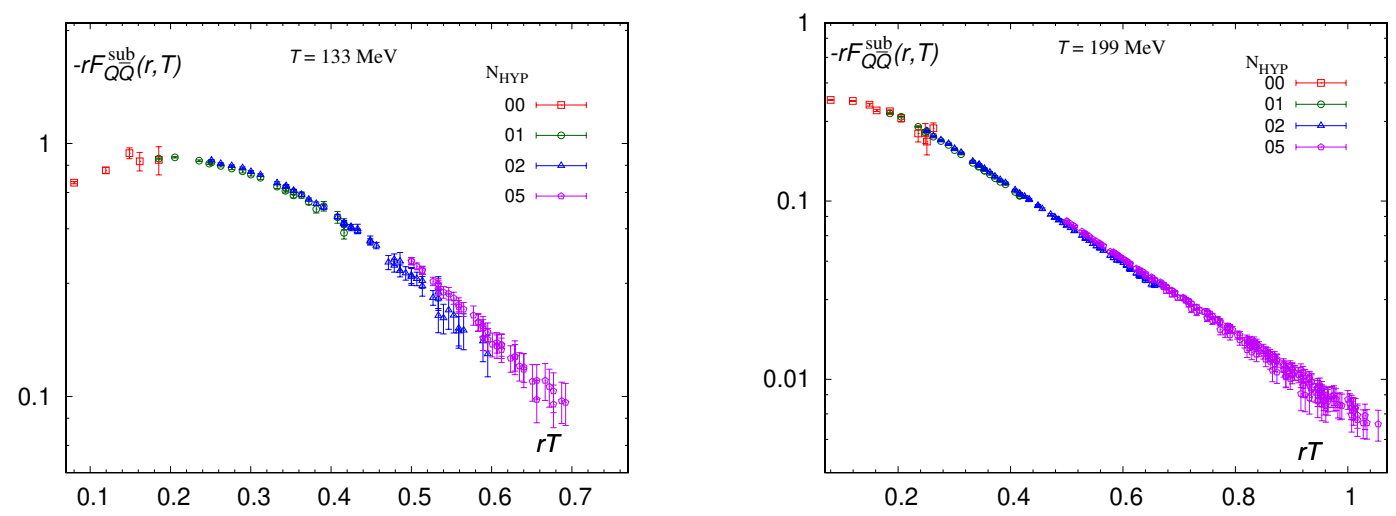

Figure 1: Screening functions for color average free energies. We show results for $N_{\tau}=12$ below $T_{c}$ (left panel) and above $T_{c}$ (right panel) for different HYP-smearing levels. These blend nicely into each other and, as discussed below, this allows us to produce a screening function spanning a large $r T$ range.

In the previous study [1] it was shown that data with $N_{\tau}=12$ is only marginally different from the continuum limit already. We use these as a proxy for a full continuum limit in the following. Multiplying the subtracted free energies for the color singlet and color average in units of the temperature $T$ by $-r$, we obtain the respective dimensionless screening functions assuming one-particle exchange. In Fig. 1 we show results for different HYP-smearing levels for two temperatures below and above $T_{c}$, respectively, for $N_{\tau}=12$. It is remarkable how well data at different smearing levels blend into one another allowing us to reach $r T$ regions well above $r T \sim 0.4$ where color screening is supposed to set in. For $T<T_{c}$ the noise exceeds the unsmeared signal already before the screening is unambiguously visible. Smearing allows us to piece together a combined screening function covering a substantially larger $r T$ range than in the earlier analysis [1], especially for $T \lesssim 2 T_{c}$. These aggregate screening functions are shown in Fig. 2, where we show $-r F_{\mathrm{S}}^{\text {sub. }}$ and $-r F_{Q \bar{Q}}^{\text {sub. }}$ with $N_{\tau}=12$ over a large temperature range. Both should appear as straight lines in the log-plot for naive exponential screening $\propto \exp (-m r)$. This is indeed the case, indicat-

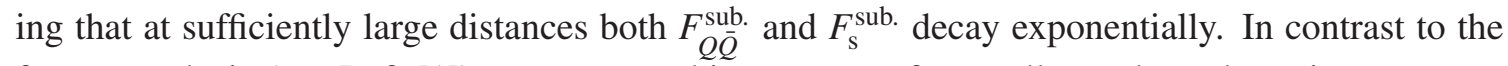
former analysis (see Ref. [1]), we can see this now even for small $T$, where the noise outgrows the signal without smearing before the exponential decay even sets in. HYP-smearing allows us to extend the signal up to $r T \sim 0.8$ in order to see and also fit the exponential decay.

As a general feature we see that the color singlet screening function gets steeper as $r T$ increases but flatter with increasing $T$. On the other hand the color average screening function changes its short distance behavior before entering the screening regime. This is due to the change from a 

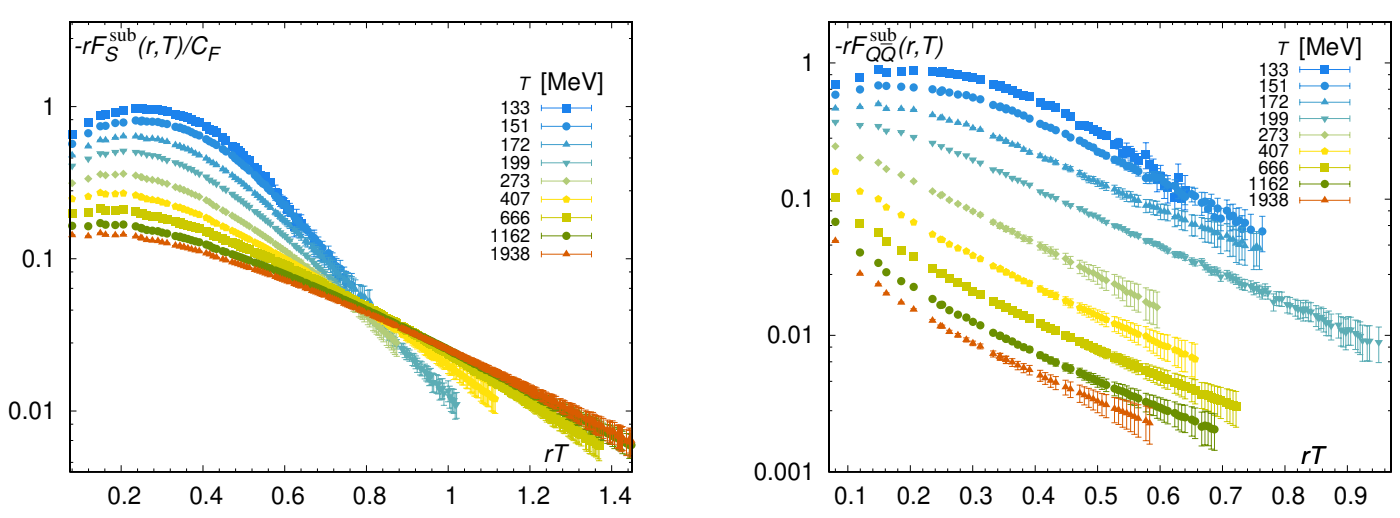

Figure 2: Screening functions for the color singlet (left panels) and the color average free energies (right panels). We show results for $N_{\tau}=12$ for a large temperature range.

Coulombic behavior in the regime $T \ll \alpha_{\mathrm{s}} / r$ to a $1 / r^{2}$ behavior in the regime $T \gg \alpha_{\mathrm{s}} / r$, which can be understood as due to cancellation between color singlet and color octet contributions as seen in weak coupling calculations. For the screening regime, we find that the color average screening function does not exhibit distinct features, i.e., the slope does not change significantly with increasing $T$ or $r T$. The leading order result for the color average free energy in the color electric screening regime is given by the exchange of two electro-static gluons (see Refs. $[8,9,10]$ ):

$$
F_{Q \bar{Q}}^{\text {sub. }} \simeq-\frac{\alpha_{\mathrm{s}}^{2}}{r^{2} T} \exp \left(-2 m_{\mathrm{D}} r\right)
$$

The perturbative NLO Debye mass (see Ref. [7]) in temperature units is given by:

$$
\begin{aligned}
\left.m_{\mathrm{D}}\right|_{\mathrm{LO}}(\mu)= & g(\mu) T \sqrt{\frac{2 N_{\mathrm{c}}+N_{\mathrm{f}}}{6}}, \\
\left.m_{\mathrm{D}}^{2}\right|_{\mathrm{NLO}}(\mu)= & \left.m_{\mathrm{D}}^{2}\right|_{\mathrm{LO}}(\mu)\left(1+\frac{\alpha_{\mathrm{s}}(\mu)}{4 \pi}\left[2 \beta_{0}\left(\gamma_{\mathrm{E}}+\ln \frac{\mu}{4 \pi T}\right)\right.\right. \\
& \left.\left.+\frac{5 N_{\mathrm{c}}}{3}+\frac{2 N_{\mathrm{f}}}{3}(1-4 \ln 2)\right]\right)-C_{\mathrm{F}} N_{\mathrm{f}} \alpha_{\mathrm{s}}^{2}(\mu) T^{2} .
\end{aligned}
$$

In the same regime of color electric screening, $r \sim 1 / m_{\mathrm{D}}$, also the color singlet free energy can be described by EQCD (See Eq. (21) in Ref. [1] for full formulae). A comparison between both screening functions and EQCD predictions using the NLO Debye mass given in Eq. (2.2) is shown in Fig. 3. The band reflects the variation of the scale $\mu$ between $\pi T$ and $4 \pi T$.

At larger distances $r \gg 1 / m_{\mathrm{D}}$, contributions from the magnetic scale $\propto g^{2} T$ may be relevant and, thus, a non-perturbative calculation is required. We expect an asymptotic screening behavior as $F^{\text {sub. }} \sim \exp (-m r) / r$. For $F_{Q \bar{Q}}$, this is due to the exchange of a single bound state of electric gluons, which may mix with contributions from the magnetic sector. We expect a similar behavior for the color singlet free energy. Comparing the $N_{\tau}=12$ results for $F_{Q \bar{Q}}$ that are shown in the Figs. 2 and 3 , we see that mainly due to the insensitivity of the screening function to changes in $r T$, a distinction between the color electric screening regime and the asymptotic screening regime is difficult. This should manifest itself in a nearly $r T$ independent screening mass. The EQCD prediction contains higher order corrections due to the running coupling that are numerically important and can be 

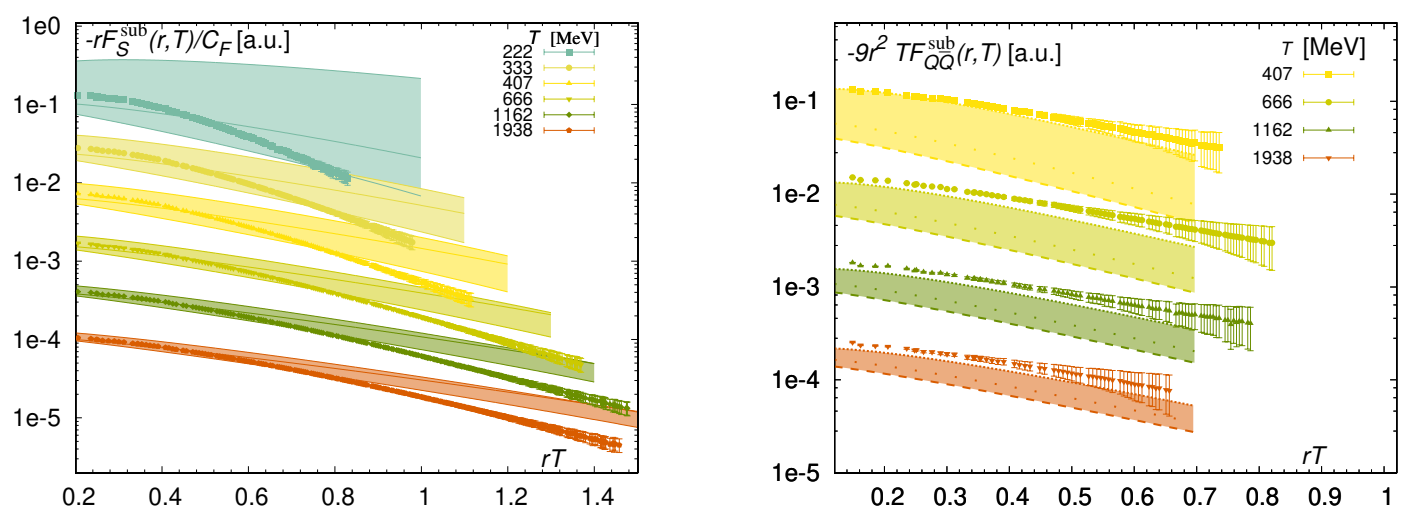

Figure 3: Comparison of the screening functions for the color singlet (left panel) and the color average free energies (right panel) to EQCD predictions (bands) at NLO. The widths of the bands correspond to the variation of the scale $\mu$ between $\pi T$ and $4 \pi T$. We show results for $N_{\tau}=12$. The results for different $T$ have been vertically displaced for better visibility.

seen in the data. The picture for the color singlet is different, as we see the increase in slope for increasing $r T$. This signals the onset of the asymptotic screening regime. We expect a significant $r T$ dependence of the screening mass.

\section{Temperature dependence of the color singlet and color average screening mass}

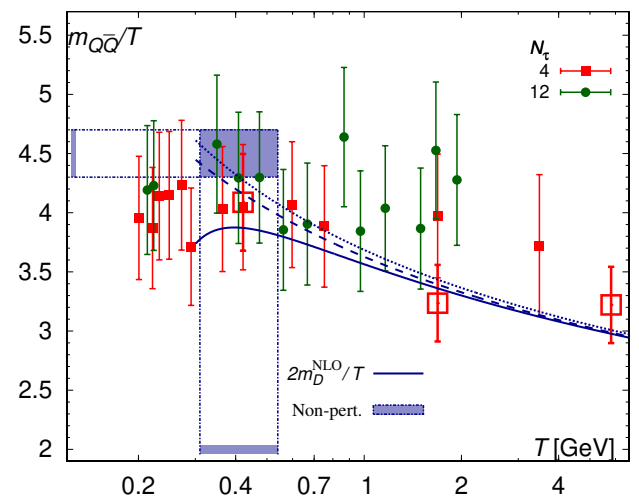

Figure 4: Color average asymptotic screening masses obtained from the exponential fits to the subtracted screening functions. The lines correspond to two times the NLO Debye mass in temperature units which is calculated for $\mu=\pi T, 2 \pi T$, and $4 \pi T$ (solid, dotted and dashed lines). The red open squares correspond to the screening masses using $N_{\tau}=4$ lattices with aspect ratio 6 . The horizontal band in the right panel corresponds to the EQCD result for the screening mass obtained in $3 \mathrm{~d}$ lattice calculations from Ref. [11].

We perform several different fits of the screening functions in order to obtain screening masses. The generic fit function for both the color singlet and color average screening function is of the form

$$
-R F=(A \exp (-M R)+c R) \Theta\left(R-i / N_{\tau}\right) .
$$

where $M=m / T, R=r T$, and $F$ is given in units of $T$. The fit parameters are $A, M$, and $c$, where for the subtracted free energies $c$ is supposedly zero in the infinite volume limit, but may actually be non-negligible due to finite volume effects or improper cancellation for even moderate statistics. In order to take into account that HYP-smearing distorts UV physics, we demand the minimal value of $R$ to be at least the number of smearing iterations divided by $N_{\tau}$. This is ensured by the Heaviside step function. For $N_{\tau}=4$ with aspect ratio 4 we consider $0,1,2$, and 3 HYP-smearing 
iterations, i.e. $i \in\{0,1,2,3\}$, and for $N_{\tau} \in\{6,8,10,12,16\}$ and for $N_{\tau}=4$ with aspect ratio 6 we additionally consider 5 HYP-smearing iterations, i.e. $i \in\{0,1,2,3,5\}$. We determine for each ensemble and HYP-smearing iteration a sensible maximal value for $R$, beyond which we no longer see exponential decay or the uncertainties of the data become too large. Within these bounds of $R$ we vary $R_{\min }$.

In a first approach we treat all the different ensembles and all smearing iterations on each ensemble as independent and perform the fits on the Jackknife averages of our data. This approach, however, lacks a handle on systematic uncertainties. In order to overcome this we perform individual fits according to Eq. (3.1) on the Jackknife bins of our data.

Figure 4 shows the asymptotic color average screening mass $m_{Q \bar{Q}} / T$ in temperature units obtained at $r T=0.5$ from the exponential fits of the screening function performed on the Jackknife bins. The results have been shifted by -0.25 with a systematic error estimate of \pm 0.1 . The red open squares are at $r T=1.3$ and correspond to the screening masses using $N_{\tau}=4$ lattices with aspect ratio 6. The lines correspond to twice the EQCD prediction, Eq. (2.2), for $\mu=\pi T, 2 \pi T$ and $4 \pi T$. The similarity of the asymptotic mass $m_{Q \bar{Q}}$ to the electric screening mass in EQCD $2 m_{\mathrm{D}}$ makes a distinction between the two regimes for $F_{Q \bar{Q}}$ particularly difficult. Our result is in good agreement with a lattice determination of the EQCD screening mass (see Ref. [11]), shown as a horizontal band.

For the color singlet (not shown) we are able to obtain results at $r T=1$. Using a $N_{\tau}=4$ aspect ratio 6 determination at $r T=1.3$, we need to shift the $r T=1$ results by +0.15 with a systematic error estimate of \pm 0.05 in order to be consistent with the previous results. The corresponding EQCD prediction, Eq. (2.2), for $\mu=\pi T, 2 \pi T$ and $4 \pi T$, requires a rescaling by a constant $A=1.6-2.0$. We then obtain that for $T \gtrsim 400 \mathrm{MeV}$ the screening mass and the rescaled Debye mass are very similar.

\section{Temperature dependence of asymptotic screening masses}
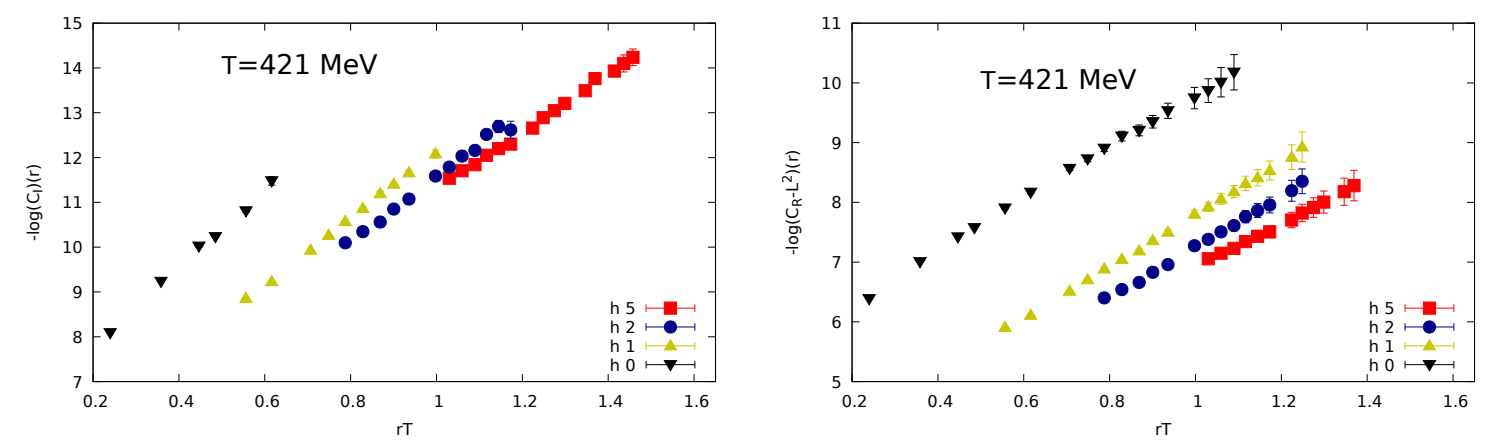

Figure 5: Imaginary (left panel) and subtracted real part (right panel) of the Polyakov loop correlator as a function of $r T$. We show a high statistics result for $N_{\tau}=4$ with aspect ratio 6 for different HYP-smearing levels.

EQCD predicts that the lowest states contributing to the correlation function of the real and imaginary part of the Polyakov loop correlator are bound states of 2 and 3 gluons, respectively. The 
corresponding screening masses, $m_{R} / T$, and $m_{I} / T$ in the asymptotic regime should then scale like

$$
\frac{m_{R}}{m_{I}} \sim \frac{2}{3}
$$

This scaling behavior has been seen on the lattice in Refs. $[12,13]$. The corresponding correlators of the real and imaginary parts of the Polyakov loop, respectively, read

$$
C_{R}(r)=\langle\mathfrak{R} P(0) \Re P(r)\rangle, \quad C_{I}(r)=\langle\mathfrak{I} P(0) \mathfrak{I} P(r)\rangle .
$$

We use the correlation functions with smeared fields and fit $C_{R, I} \sim \exp \left(-m_{R, I} r\right) /(r T)+$ const. to probe the mass of the lowest energy state that is exchanged and compare these masses to the weakcoupling prediction of EQCD. We show an example of the correlators in Fig. 5 as a function of $r T$ for $N_{\tau}=4$ with aspect ratio 6 for different HYP-smearing levels. Since the Polyakov loop expectation value is real in QCD, $C_{R}$ asymptotes to $L^{2}$, which we can subtract, while the imaginary part approaches zero asymptotically. In contrast to the color average and color singlet screening functions it is not possible to piece together one correlator for the real and imaginary parts of the Polyakov loop correlator without changing the normalization by hand. For aspect ratio 4 ensembles the $\mathrm{S} / \mathrm{N}$ is usually significantly worse for both correlators. The imaginary part correlator has a worse $\mathrm{S} / \mathrm{N}$ ratio but the real part correlator suffers from thermal IR noise due to the subtraction. This can be seen when, e.g., comparing the unsmeared results (black symbols).

This in general makes it quite difficult to extract screening masses and even more complicated to determine their ratio. Our preliminary results on a few ensembles with high statistics are reasonably consistent with the EQCD prediction and with Ref. [13], i.e., we obtain as our most precise result for the ensemble of Fig. $5 m_{I} / m_{R} \approx 1.72(4), m_{R} / T \approx 4.3(1)$, and $m_{I} / T \approx 7.4(2)$. Both masses tend to decrease at higher temperatures; $m_{I} / T$ appears to decrease more strongly.

From our results with aspect ratio 6 we conclude that $m_{I} / m_{R}$ can be determined with about $10 \%$ accuracy at $r T \gtrsim 0.6$, while smaller distances are contaminated by physics of the electric screening regime.

\section{Summary}

We have performed studies of quark-antiquark correlation functions covering a wide range of temperatures from below to far above $T_{c}$. Making use of HYP-smearing we have been able to obtain a signal for color singlet and color average free energies in the screening regime at $T<T_{c}$ and extract corresponding screening masses. In comparison with EQCD predictions for the screening masses, we obtain reasonable agreement for $T \gtrsim 400 \mathrm{MeV}$.

A second prediction of EQCD is the ratio of the screening masses of the real and imaginary part of the Polyakov loop correlator, which we see reasonably well confirmed in our measurement for high $T$, although this analysis is still ongoing.

At present all of our results are preliminary, as they lack continuum extrapolation. The full results will be discussed in a future publication [2]. 


\section{Acknowledgments}

This research was supported by the DFG cluster of excellence "Origin and Structure of the Universe" (www.universe-cluster.de). The simulations have been carried out on the computing facilities of the Computational Center for Particle and Astrophysics (C2PAP) and SuperMUC using the publicly available MILC code. This work has been supported in part by the U.S Department of Energy through grant contract No. DE-SC0012704.

The authors would like to thank P. Petreczky for numerous discussions and support.

S.S. would like to thank N. Brambilla and A. Vairo for support, and TUMGlobal for financial support and MSU and BNL for hospitality during a visit.

\section{References}

[1] A. Bazavov, N. Brambilla, P. Petreczky, A. Vairo and J. H. Weber, Color screening in $2+1$ flavor QCD, Phys. Rev. D98 (2018) 054511 [1804.10600].

[2] TUMQCD Coll., in preparation: TUM-EFT 120/18.

[3] M. Berwein, N. Brambilla, P. Petreczky and A. Vairo, The Polyakov loop correlator in perturbation theory, Phys. Rev. D96 (2017) 014025 [170 . 07266].

[4] A. Hasenfratz and F. Knechtli, Flavor Symmetry and the Static Potential with Hypercubic Blocking, Phys. Rev. D64 (2001) 034504 [0103029].

[5] E. Follana, Q. Mason, C. Davies, K. Hornbostel, G. P. Lepage, J. Shigemitsu et al., Highly Improved Staggered Quarks on the Lattice, with Applications to Charm Physics, Phys. Rev. D75 (2007) 054502 [0610092].

[6] A. Bazavov, C. Bernard, C. DeTar, W. Freeman, S. Gottlieb, U. M. Heller et al., Scaling studies of QCD with the dynamical HISQ action, Phys. Rev. D82 (2010) 074501 [1004.0342].

[7] E. Braaten and A. Nieto, Free Energy of QCD at High Temperature, Phys. Rev. D53 (1996) 3421 [9510408].

[8] D. J. Gross, R. D. Pisarski and L. G. Yaffe, QCD and Instantons at Finite Temperature, Rev. Mod. Phys. 53 (1981) 43.

[9] L. D. McLerran and B. Svetitsky, Quark Liberation at High Temperature: A Monte Carlo Study of SU(2) Gauge Theory, Phys. Rev. D24 (1981) 450.

[10] S. Nadkarni, Nonabelian Debye Screening. 1. The Color Averaged Potential, Phys. Rev. D33 (1986) 3738.

[11] A. Hart, M. Laine and O. Philipsen, Static correlation lengths in QCD at high temperatures and finite densities, Nucl. Phys. B586 (2000) 443 [0 004060 ].

[12] S. Datta and S. Gupta, Does the QCD plasma contain propagating gluons?, Phys. Rev. D67 (2003) 054503 [0208001].

[13] S. Borsányi, Z. Fodor, S. D. Katz, A. Pásztor, K. K. Szabó and C. Török, Static $\bar{Q} Q$ pair free energy and screening masses from correlators of Polyakov loops: continuum extrapolated lattice results at the QCD physical point, JHEP 04 (2015) 138 [1501.02173]. 